\title{
Flexible Video-Endsocopic Injection Sclerotherapy for Second and Third Degree Internal Hemorrhoids
}

\author{
Sandeep Nijhawan, Udawat H, Gaurav Gupta, Anil Sharma, Amit Mathur, Bharat Sapra, Subhash \\ Nepalia
}

Department of Gastroenterology, SMS Medical College, Jaipur, Rajasthan, India

\section{ABSTRACT}

\begin{abstract}
Background and objectives: Bleeding from hemorrhoids is the commonest cause of rectal bleeding in adults. Injection sclerotherapy of internal hemorrhoids is one of the non-surgical treatments, and is simple, safe and feasible. Conventionally sclerotherapy is performed with rigid proctoscope which has limitations of maneuverability, narrower field of vision and documentation compared to flexible videoendoscope. Therefore, we assessed the efficacy and safety of video-colonoscopic sclerotherapy for bleeding internal hemorrhides.
\end{abstract}

Methods: Seventy-nine patients of bleeding internal hemorrhoids were subjected to colonoscopic sclerotherapy using $1.5 \%$ polidocanol in retroflexed or forward viewing positions. Success of treatment was defined as cessation of bleeding for six weeks. Patients were observed for complications and were followed up regularly for 3 months.

Results: A total of 79 evaluable patients, 61 had grade II and 18 had grade III hemorrhoids. There was no statistically significant differences in achieving excellent or good results for control of bleeding between patients with grade II and grade III hemorrhoids $(100 \%$ vs $94,5 \% ; p>0.05)$. The number of sessions of sclerotherapy required were significantly more in grade II than grade III hemorrhoids $(1.1 \pm 0.3$ vs $1.3 \pm 0.7 ; p=0.04)$. No significant complications were noted except for bloating in ten patients $(12.6 \%)$ and rectal pain in $6(7.6 \%)$ patients. Median time taken for the procedure was 30 minutes.

Conclusions: Video-endoscopic sclerotherapy is safe, well-tolerated and effective treatment for bleeding internal hemorrhoids. (JDigEndosc 2011;2(1):1-5)

Keywords: Hematochezia-Sclerosants-Polidocanol-Hemorrhoides-Sclerotherapy-Colonoscopy

\section{Introduction}

Internal hemorrhoids are the commonest cause of rectal bleeding in adults. The treatment of hemorrhoidal disease needs to be tailored according to the grade of hemorrhoids, patient preference, and availability and expertise of the procedure. Broadly, grade I and II hemorrhoids are treated with non-operative treatment in the form of dietary modification, injection sclerotherapy, rubber band ligation, endoscopic band ligation, electrocoagulation (bipolar diathermy and direct-current electrotherapy) or infrared coagulation. [1-3] For grade III hemorrhoids modalities like banding, Doppler guided hemorrhoidal artery ligation or surgery are available. However, surgery is the treatment of choice for grade IV hemorrhoids and complicated hemorrhoids.[1-3]

Primary goal of all forms of therapy is to achieve fibrosis and obliteration of the bleeding vessel. Sclerotherapy is one of the oldest, commonly practiced and easily available forms

Reprints requests and correspondence:

Sandeep Nijhawan, MD, DM

Department of Gastroenterology,

SMS Medical College, Jaipur, Rajasthan, India

Dr_nijhawan@yahoo.com 
of nonsurgical treatment. The proposed mechanisms of sclerotherapy are thrombosis of vessels, sclerosis of the connective tissue, sub mucosal fibrosis, shrinkage and fixation of the overlying mucosa.[3] Conventionally, it is performed using a rigid proctoscope, long injector needle such as lumbar puncture needle and a sclerosant $(5 \%$ phenol in almond oil, $5 \%$ quinine and urea or $23.4 \%$ hypertonic saline) injected at the base of hemorrhoidal complex. [4] In contrast to the flexible video endoscope, the rigid proctoscope has limited maneuverability, has a narrower field of view, leads to more discomfort at introduction and does not allow adequate documentation. [5] Also, the knee-chest position is uncomfortable and awkward for many patients. To overcome these drawbacks of rigid proctoscope and advantages of preciseness of endoscopic vision and guided therapeutic procedures; and considering the ease, simplicity and effectiveness of endoscopic polidocanol injection in esophageal varices; we combined the two modalities and came up with an idea of studying the efficacy and safety of injection of grade II and III hemorrhoids with the help of a colonoscopic-guided injection with a long sclerotherapy needle using polidocanol solution.

\section{Methods}

This was a prospective study carried out at a tertiary care centre in Western India over a period of nine months. Grading of hemorrhoids was done using classification proposed by Banov et al.[10] The study was approved by institutional ethics committee, and written informed consent was taken from every patient.

\section{Inclusion and exclusion criteria}

The study group included patients with bleeding grade II and III internal hemorrhoids. Patients without any significant co-morbid illness were included in the study. Exclusion criterion for the study protocol were patients with external hemorrhoids, rectal prolapse, grade IV hemorrhoids, rectal polyp, and anal fissure and thrombosed hemorrhoids.

\section{Technique of colonoscopic sclerotherapy}

Initially all the patients underwent ileocolonoscopy with flexible video-colonoscope (Olympus Evis Smartage CFV 70 L) after a proper colon preparation with polyethylene glycol lavage solution, under conscious sedation using intravenous injection of midazolam to exclude other source of rectal bleeding. In every patient after returning to the rectum, the colonoscope was retroflexed by turning the tip of the instrument upward and simultaneously, gently advancing and torquing the endoscope in counter-clockwise fashion. This was done to have a clear view of the dentate line, hemorrhoids and relationship of hemorrhoids to the dentate line. The characteristics of each hemorrhoidal column like size, breach in the mucosa over the hemorrhoids and the continuation of a venous channel proximal to the hemorrhoids was noted in all patients. The site of injection chosen was either a prominent venous channel in continuation with the hemorrhoid proximally or the hemorrhoid itself taking precaution that the site of injection was proximal to the dentate line so as to ensure painless injection. The colonoscopic sclerotherapy (CST) was performed in both retroflexed and forward viewing positions of the scope. Figure 1 shows the diagrammatic representation

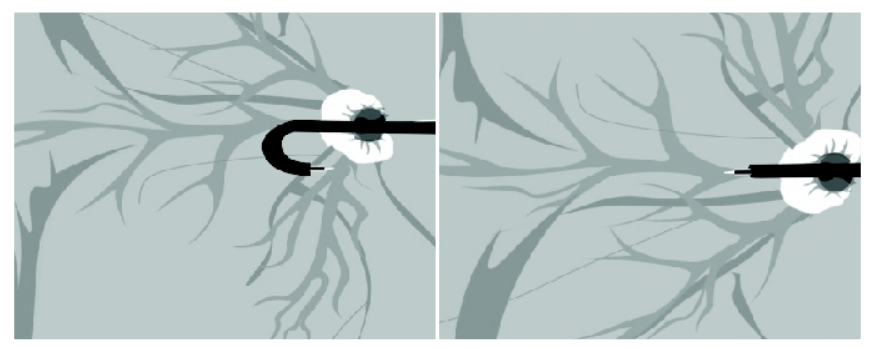

Figure 1: Schematic representation of the two methods used for colonoscopic sclerotherapy of hemorrhoidal complex. Top image shows retrograde injection and bottom image show ante grade injection

of the method for CST. A standard endoscopic injection catheter with $23 \mathrm{G}$, 5-mm long retractable needle was introduced through the biopsy channel to the tip while viewing the mid-rectum in antegrade fashion. Hemorrhoidal complexes were then viewed from above and the endoscope maneuvered to facilitate precise placement of the injection needle in the proximal portion of the hemorrhoidal column above the dentate line. Care was taken to avoid excessive torque or looping of the endoscope to prevent difficulties with extension of the endoscopic injection needle. The injection catheter was then preflushed with the diluted sclerosant, the needle extended, and injections commenced; $0.5 \mathrm{ml}$ of $1.5 \%$ polidocanol was injected proximal to the dentate line (Askerol, Samarth Pharmacy Private Limited, India). The end point of each injection was to achieve complete blanching of the column. Appropriate injection was confirmed by ease of injection and recognition of dissemination of the sclerosant in the hemorrhoidal complex. Once appropriate placement was apparent, full volume of sclerosant was slowly delivered. Procedure was stopped whenever patient had pain during injection and technique was revised such as to achieve ideal completion. Ante grade injections were given if the columns were short and difficult to reach on retroflexion. Injections were performed in all columns of hemorrhoids by rotating the scope in anal canal and injecting proximal to the dentate line. The method of injection of hemorrhoids using a colonoscope, a sclerotherapy injector and polidocanol was named as colonoscopic sclerotherapy (CST) of hemorrhoids. Instances of minor post-injection bleeding were treated by simple observation. At the end of the 
procedure insufflated air was suctioned so as to reduce abdominal distension. Patients were advised Sitz bath at night if they experienced perianal itching, pain and burning.

\section{Follow-up}

The patients were followed at 2 weeks interval for 1 month and thereafter every month, till the end of the study. Patients were subjected to a repeat procedure if the first session of CST did not achieve the desired response i.e. cessation of bleeding at 2 weeks. Success of the treatment was defined as cessation of bleed for 6 weeks. The response was graded as excellent (complete cessation of bleed per rectum), good (definite improvement but occasional bleed per rectum) and poor (no improvement in bleeding per rectum).[5] Pain after CST was graded as mild (mild rectal discomfort, tenesmus, or both requiring no analgesic medication) or severe (requiring oral or injectable analgesics).

\section{Statistical analysis}

For data management and statistical analysis, SPSS-10 software (SPSS Inc., Chicago, IL) was used. All quantitative data was expressed as mean with standard deviation and compared with the Student $t$ test. A $p$ value of less than 0.05 was considered significant.

\section{Results}

During the study period a total of 114 patients with bleeding per rectum were enrolled. Eighty-five patients of internal hemorrhoids fulfilled the inclusion criteria and were subjected to colonoscopic sclerotherapy. There were 51 males and 34 females. None of these patients had another source of bleeding. Out of 85 patients, six patients were lost to follow-up. Finally, 79 patients completed the study and were included for final analysis. The baseline data of these 79 patients is shown in Table 1. There were 6 patients of ischemic heart disease on aspirin and clopidogrel and two of them (2.5\% of total number of patients) required three units

Table 1: Baseline data of 79 patients

$\begin{array}{lc}\text { Age in years, mean } \pm \text { SD } & 55.4 \pm 10.2 \\ \text { Gender, male:female } & 47: 32 \\ \text { Hemorrhoids, grade 2:3 } & 61: 18 \\ \text { Bleeding } & 79 \\ \text { Hemoglobin gm/100 ml, mean } \pm \text { SD } & 10.8 \pm 1.4 \\ \text { Duration of symptoms in days, mean } \pm \text { SD } & 40.8 \pm 35.7 \\ \text { Previous treatment for hemorrhoids } & \\ \quad \text { Hemorrhoidectomy } & 2 \\ \text { Sclerotherapy (Proctoscope guided) } & 15 \\ \text { Band ligation } & 12 \\ \text { Injection therapy by a non-medical person } & 10 \\ \text { Treatment naive } & 40\end{array}$

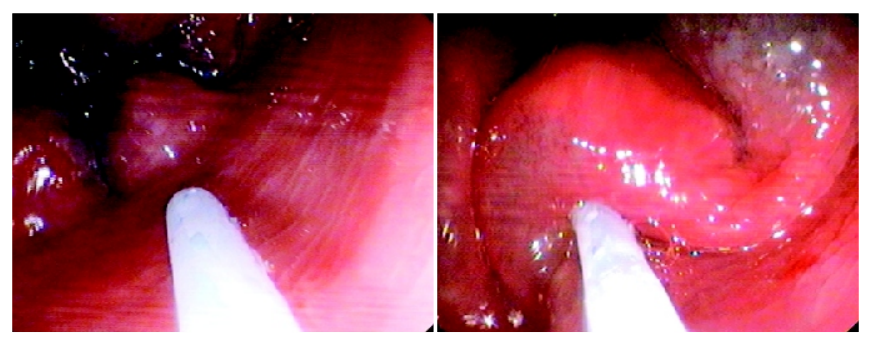

Figure 2: The columns of hemorrhoids seen endoscopically in a retroverted position with the injector catheter in view. Image on the right side shows polidocanol being injected at the base of the hemorrhoids.

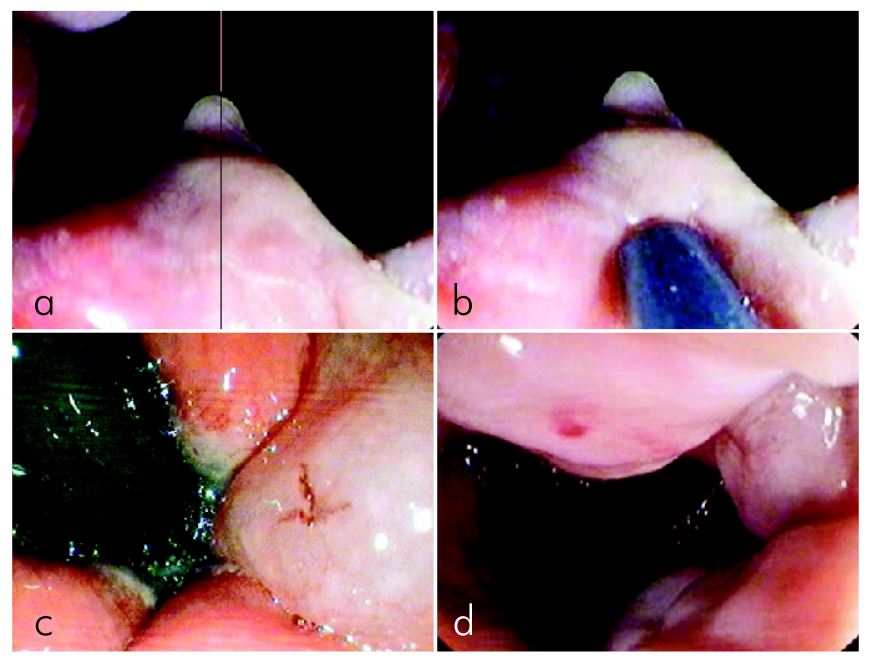

Figure 3: (a)The hemorrhoids in forward viewing position of colonoscope, (b) injection of polidocanol in the same position, (c) Immediately after injection the marks of injection can be seen in retroverted and (d) forward viewing position

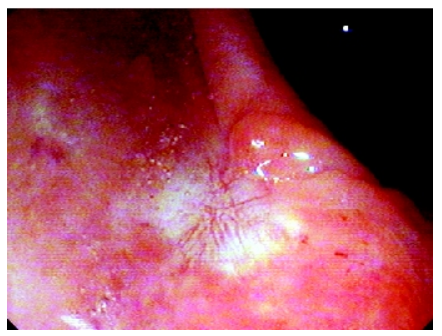

Figure 4: Cicatrisation at the site of injection of polidocanol seen during the second sitting of sclerotherapy and

disappearance of hemorrhoids.

of packed cell transfusion for severe anemia due to bleeding. Sclerotherapy was done with retroflexed view (Figure 2) in $63(79.7 \%)$ patients and in forward viewing position (Figure $3)$ in 16 patients $(20.3 \%)$.

The efficacy of sclerotherapy to stop bleeding is shown in Table 2. There was significant difference in number of sessions required in patients of grade II and grade III hemorrhoids $(1.1 \pm 0.3$ vs $1.3 \pm 0.7 ; p=0.04)$. Table 3 shows that majority of patients in both with grade II and grade III hemorrhoids required a single session. Only 1 patient with grade III hemorrhoids needed three sessions. Cicatrisation after sclerotherapy was observed (Figure 4) at the injection 
Table 2: Results of colonoscopic sclerotherapy of hemorrhoids

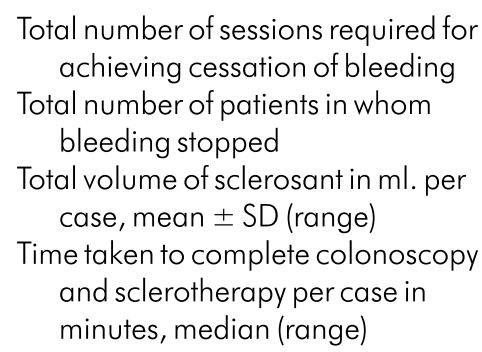

$6.4 \pm 2.2,(4-12)$

$30(25-50)$

Table 3: Number of sessions of flexible video-endoscopic sclerotherapy required for cessation of bleed

\begin{tabular}{lcc} 
No. of sessions & $\begin{array}{c}\text { Grade II } \\
(\mathrm{n}=61)\end{array}$ & $\begin{array}{c}\text { Grade III } \\
(\mathrm{n}=18)\end{array}$ \\
One & $54(88.5 \%)$ & $13(72.2 \%)$ \\
Two & $7(11.4 \%)$ & $4(22.2 \%)$ \\
Three & - & $1(5.5 \%)$ \\
\hline Total & 68 & 24
\end{tabular}

Table 4: Comparison of cessation of bleeding in different grades of hemorrhoids

\begin{tabular}{lccc} 
Type of response & $\begin{array}{c}\text { Grade II } \\
61 \text { cases }\end{array}$ & $\begin{array}{c}\text { Grade III } \\
18 \text { case }\end{array}$ & pvalue \\
Excellent & $49(80.3 \%)$ & $12(66.7 \%)$ & 0.33 \\
Good & $12(19.7 \%)$ & $05(27.8 \%)$ & 0.72 \\
Poor & $0(0 \%)$ & $1(5.5 \%)$ & 0 \\
\hline
\end{tabular}

sites during the second and subsequent session of sclerotherapy in $25 \%$ of patients. Table 4 shows that there was no significant difference in terms of excellent and good responses between patients with grade II and III hemorrhoids (100\% vs $94.5 \%)$.

\section{Complications}

Ten patients (12.7\%) developed bloating during the procedure due to continuous insufflation of air, which improved after air was suctioned out. Six patients $(7.6 \%)$ developed mild post procedural rectal pain. There were no local complications in form of rectal or perianal abscess, urinary retention, dysuria, strangury or impotence. There was no systemic complication in form of portal pylephlebitis, portal pyaemia, septicemia and embolism.

\section{Discussion}

The present study has shown that CST with polidocanol achieved good long term results in stopping bleeding in grade II and III hemorrhoids. The study did not look into other parameters like erdication of hemorrhoids, disappearance of prolapse or pain. The maximum number of sessions required to stop hemorrhoidal bleed was two sessions in grade II hemorrhoids and three sessions in grade III hemorrhoids. Majority of patients responded to a single session of CST in both the grades of hemorrhoids (88\% in grade II and $72 \%$ in grade III). Although both grades of hemorrhoids responded well to CST, the excellent and good responses were statistically more significant in grade II hemorrhoids than grade III hemorrhoids. The reason for overall good response with colonoscopic sclerotherapy was probably the preciseness of the injections into the hemorrhoidal complex guided by the vision of colonoscope and effectiveness of polidocanol as a sclerosant. The chances of deep transmucosal injections and excess amount being injected are expected to be lower. Comparison with other established procedures was not done. However, the colonoscope is not widely available and hence this technique may not find wide applicability in comparison to a proctoscope.

The CST was a safe procedure and none experienced side effects that have been reported with conventional sclerotherapy of hemorrhoids. Abdominal distention as a result of air insufflation in colonoscopy is transient and could be reduced by proper suctioning of air in the rectum.

The data on the endoscopic injection sclerotherapy of internal hemorrhoids is sparse.[11-15] Ponsky et al reported an initial evaluation of $23.4 \%$ saline in 19 patients with symptomatic grade I, II, or III hemorrhoids. The technique was reported to be very effective in relieving bleeding and alleviating prolapse. The technique was well tolerated and associated with high patient satisfaction and low complication rates, with no serious complications. ${ }^{11}$ Benin et al treated 250 patients of grade II-IV hemorrhoids with sotradecol foam. Bleeding and hemorrhoidal prolapse were resolved with at most two sclerotherapy sessions. However, pain usually had disappeared after the first session. No complications such as mucosal erosions at the foam injection site, abscesses formation, bacteremia, or fistulas occurred. ${ }^{12}$ Alatise et al concluded that endoscopic hemorrhoidal sclerotherapy using $50 \%$ dextrose water was a simple, safe and effective modality of treatment in 40 patients and controlled bleed in all patients. [15]

Since sclerotherapy was done in one sitting immediately after completion of colonscopy and conventional sclerotherapy equipment was used, this approach may reduce work-load on endoscopy staff and cost of the procedure. Sclerotherapy in retroflexed position was favored in the present study over the forward-viewing approach because of ease of injection. The procedure is well tolerated and safe. Only 10 patients developed feeling of bloating during the procedure, which improved after suction of the insufflated air. Six $(7.6 \%)$ patients had mild rectal pain, which improved spontaneously. The complications in our study were comparable to the band ligation. Flexible video-endoscopic sclerotherapy is very effective as it improved the symptoms in majority of the patients.

Greater efficacy of endoscopic guided procedures has 
been proven with endoscopic band ligation of hemorrhoids. When video endoscopic elastic band ligation was performed, a significantly lower number of treatment sessions was required in the video endoscopic elastic band ligation group (1.8 [0.8] vs. 2.4 [0.9]; $p<0.01)$ and the total number of bands applied was significantly less (2.8 [1.1] vs. 3.7 [1.4]; $p<$ 0.01).[16] There is no data to compare flexible videoendoscopic sclerotherapy with conventional sclerotherapy.

There are studies that have compared conventional injection sclerotherapy with other modalities in treatment of hemorrhoids but not endoscopic sclerotherapy with other modalities. In a study comparing conventional injection sclerotherapy and surgery in patients with third- and fourthdegree internal hemorrhoids at 28 days after treatment, the disappearance rate of prolapse was similar between injection sclerotherapy and surgery, 94\% (75/80 patients) and 99\% (84/85 patients), respectively. The 1 -year recurrence rate was $16 \%$ (12/73 patients) in the sclerotherapy group and this value was satisfactory because of its less invasive nature while it was more or less higher compared with $2 \%$ (2/81 patients) in the surgery group. The incidence of pain and bleeding were lower in the injection sclerotherapy group. [17]In yet another comparative trial band ligation (77\%) faired better than conventional injection sclerotherapy $(63.3 \%)$ but band ligation was more painful.[18]

\section{Conclusion}

In summary, flexible video-endoscopic sclerotherapy is a safe, well tolerated, and effective modality for management of bleeding internal hemorrhoids. However, further prospective randomized comparative trials with other modalities would address future issues and determine the long-term results.

\section{References}

1. Madoff RD, Fleshman JW. American Gastroenterological Association Technical Review on the diagnosis and treatment of hemorrhoids. Gastroenterology 2004;126:1463-73.

2. Steele SR, Madoff RD. Systematic review: the treatment of anal fissure. Alimentary Pharmacology \& Therapeutics 2006;24: 247-57.

3. Chong PS, Bartolo DCC. Hemorrhoids and Fissure in Ano. Gastroenterol Clin NAm 2008;37: 627-44.

4. Sim AJ, Murie JA, Mackenzie I. Three year follow-up study on the treatment of first and second-degree hemorrhoids by sclerosant injection or rubber band ligation. Surg Gynecol Obstet 1983;157:534-6.

5. Berkelhammer C, Moosvi SB. Retroflexed endoscopic band ligation of bleeding internal hemorrhoids. Gastrointest Endoscopy 2002;55:532-7.

6. MacRae HM, McLeod RS. Comparison of hemorrhoidal treatment modalities. A meta-analysis. Dis Colon Rectum 1995;38:687-94.

7. Johanson JF, Rimm A. Optimal nonsurgical treatment of hemorrhoids: a comparative analysis of infrared coagulation, rubber band ligation, and injection sclerotherapy. Am J Gastroenterol 1992;87:1600-6.

8. Senapati A, Nicholls RJ. A randomised trial to compare the results of injection sclerotherapy with a bulk laxative alone in the treatment of bleeding haemorrhoids. Int J Colorectal Dis 1988;3:124-6.

9. Su MY, Chiu CT, Wu CS, Ho YP, Lien JM, Tung SY, Chen PC. Endoscopic hemorrhoidal ligation of symptomatic internal hemorrhoids. Gastrointest Endosc 2003;58: 871-4.

10. Banov L Jr, Knoepp LF Jr, Erdman LH, Alia RT. Management of hemorrhoidal disease.J S C Med Assoc 1985;81:398-401.

11. Ponsky JL, Mellinger JD, Simon IB. Endoscopic retrograde hemorrhoid sclerotherapy using $23.4 \%$ saline: a preliminary report. Gastrointest Endosc 1991;37:155-8.

12. Benin P, D'Amico C. Foam sclerotherapy with Fibrovein (STD) for the treatment of hemorrhoids, using a flexible endoscope. Minerva Chir 2007;62:235-40.

13. Chiappone GM, Malpas PM. Endoscopic retrograde hemorrhoid sclerotherapy. Gastroenterol Nurs 1992;15:78-80.

14. Johanson JF, Rimm A. Optimal nonsurgical treatment of hemorrhoids: a comparative analysis of infrared coagulation, rubber band ligation and injection sclerotherapy. Am J Gastroenterol 1992;87:1600-6.

15. Alatise OI, Arigbabu OA, Lawal OO, Adesunkanmi AK, Agbakwuru AE, Ndububa DA, Akinola DO. Endoscopic hemorrhoidal sclerotherapy using $50 \%$ dextrose water: a preliminary report. Indian J Gastroenterol 2009;28:31-2.

16. Wehrmann T, Riphaus A, Feinstein J, Stergiou N. Hemorrhoidal elastic band ligation with flexible videoendoscopes: a prospective, randomized comparison with the conventional technique that uses rigid proctoscopes. Gastrointest Endosc 2004;60:191-5.

17. Takano M, Iwadare J, Ohba H, Takamura H, Masuda Y, Matsuo K, Kanai $\mathrm{T}$ et al. Sclerosing therapy of internal hemorrhoids with a novel sclerosing agent. Comparison with ligation and excision. Int J Colorectal Dis 2006;21:44-51.

18. Sim AJ, Murie JA, Mackenzie I. Comparison of rubber band ligation and sclerosant injection for first and second degree haemorrhoids-a prospective clinical trial. Acta Chir Scand 1981;147:717-20.

Source of support: Nil; Conflict of interest: none declared 